\title{
Pediatric tuberculosis: clinical and epidemiological reflections from a highly endemic setting
}

\author{
Anneke Hesseling \\ From Immunodiagnosis of Tuberculosis: New Questions, New Tools \\ Virginia, VA, USA. 21-23 September 2008
}

Pediatric TB provides unique opportunities to study TB disease epidemiology. Improved diagnostics are of key importance in order to address many of the challenges posed by TB in children. In the Western Cape Province in South Africa, the TB notification rate was more than 600 out of 100,000 for children aged 0-14 years in 2007; the maternal HIV prevalence was $15 \%$, with a good vertical preventative program. The HIV transmission rate was $4-5 \%$; $99 \%$ of neonates routinely receive BCG vaccination at birth. Isoniazid prophylaxis, shown to be effective in the prevention of TB in young children, is seldom initiated and many missed opportunities for preventive therapy exist. In children admitted to hospital, mycobacterial culture is routinely done for TB in children less than five years of age through gastric aspiration of stomach contents in this setting; the culture yield is $30-40 \%$ in children with clinically suspected TB.

In a recent study of infants admitted to the three Cape Town hospitals over a three-year period, $55 \%$ of children born to HIV positive mothers had a household contact with $\mathrm{TB}$, indicating a high level of $\mathrm{TB}$ exposure. A high risk of serious BCG vaccine complications exists in HIV-positive infants in the form of disseminated BCG disease, with an incidence of 992 per 100000 (95\% CI: 567-1495). More research is needed to determine if $B C G$ vaccination protects against $\mathrm{TB}$ in infants born to $\mathrm{HIV}$-infected women based on the high risk in the excessively high TB incidence (more than 20-fold higher) in HIV-infected infants.
Because scientists have improved techniques for isolating Mycobacterium tuberculosis in children and increasing the yield, it is possible to identify the strains from infected children, which can impact diagnosis and treatment. Our team has developed a protein-enriched broth that increases yield and decreases the time to detection. Pediatric TB also offers an opportunity to look at the emergence of drug resistant TB strains. In three DST surveys conducted in Cape Town (1997 through 2007) results indicated a significant increase in both drug and multi-drug resistant cases of TB in children. Examination of genotypes in 400 culture positive children showed the most prevalent form to be the Beijing strain, thought to have the greatest probability of progressing from TB infection to disease and with distinct disease forms. Programs to prevent TB and TB/HIV co-infection in children and adults must be improved; and safer vaccines, better diagnostics and shorter treatment regimens developed targeting children.

Published: 17 December 2010

doi:10.1186/1753-6561-4-S3-012

Cite this article as: Hesseling: Pediatric tuberculosis: clinical and epidemiological reflections from a highly endemic setting. $B M C$ Proceedings 2010 4(Suppl 3):012. 